\title{
LINEAR AUTOMATON TRANSFORMATIONS
}

\section{A. NERODE ${ }^{1}$}

Let $R$ be a nonempty set, let $N$ consist of all non-negative rational integers, and denote by $R^{N}$ the set of all functions on $N$ to $R$. If $R$ is a ring, a map $M: R^{N} \rightarrow R^{N}$ is linear if $M\left(r_{1} f_{1}+r_{2} f_{2}\right)=r_{1}\left(M f_{1}\right)$ $+r_{2}\left(M f_{2}\right)$ for $r_{1}, r_{2}$ in $R, f_{1}, f_{2}$ in $R^{N}$. For a finite commutative ring with unit we determine which linear transformations $M: R^{N} \rightarrow R^{N}$ can be realized by finite automata.

More precisely, let $A, B$ be finite nonempty sets. A map $M: A^{N} \rightarrow B^{N}$ is an automaton transformation if there exists a finite set $Q$, maps $M_{Q}: A \times Q \rightarrow Q, M_{B}: A \times Q \rightarrow B$, elements $\bar{b}$ in $B, \bar{q}$ in $Q$ such that corresponding to each $f$ in $A^{N}$ there exists an $h$ in $Q^{N}$ satisfying

$$
\begin{array}{cl}
h(0)=\bar{q}, & h(n+1)=M_{Q}(f(n), h(n)), \quad(M f)(0)=\bar{b}, \\
& (M f)(n+1)=M_{B}(f(n), h(n)) .
\end{array}
$$

(In automaton language, $A$ is the input alphabet, $B$ is the output alphabet, $Q$ is the set of states, $\bar{q}$ is the initial state, $\bar{b}$ is the initial output, while $M_{B}(a, q)$ and $M_{Q}(a, q)$ are respectively the output and state resulting from input $a$ and state $q$. For the case that $A$ and $B$ coincide with the set consisting of 0 and 1 , the concept of automaton transformation is simply a variant of the concept of representable event of Kleene [1].)

Call a matrix $u_{i j}: N \times N \rightarrow R$ eventually doubly-periodic if for some positive integers $P_{1}, P_{2}, p_{1}, p_{2}$ :

$$
\begin{array}{ll}
u_{i j}=u_{\left(i+p_{1}\right) j} & \text { for all } i>P_{1} \text { and all } j, \\
u_{i j}=u_{i\left(j+p_{2}\right)} & \text { for all } j>P_{2} \text { and all } i .
\end{array}
$$

ThEOREM 1. Let $R$ be a finite commutative ring with unit. Then $M: R^{N} \rightarrow R^{N}$ is a linear automaton transformation if and only if there exists a matrix $u_{i j}: N \times N \rightarrow R$ such that:

(i) for all $j, u_{0 j}=0$;

(ii) for $f$ in $R^{N}$ and $n \geqq 0,(M f)(n)=u_{n 0} f(0)+u_{(n-1) 1} f(1)+\cdots$ $+u_{0 n} f(n)$

(iii) $u_{i j}$ is eventually doubly-periodic.

Define $\tau: R^{N} \rightarrow R^{N}$ by $(\tau f)(0)=0,(\tau f)(n)=f(n-1), n \geqq 1$. A map $M: R^{N} \rightarrow R^{N}$ is translation invariant if for $f$ in $R^{N}, M \tau f=\tau M f$. Call a sequence $u_{0}, u_{1}, \cdots$ eventually periodic if there exist positive integers 1957.

Presented to the Society January 30,1958; received by the editors December 26,

1 National Science Foundation postdoctoral fellow. 
$P, p$ such that $u_{n+p}=u_{n}$ for $n \geqq P$, then $p$ is a period.

Corollary. Let $R$ be a finite commutative ring with unit. Then $M: R^{N} \rightarrow R^{N}$ is a linear translation invariant automaton transformation if and only if there exists an eventually periodic sequence $u_{0}=0, u_{1}$, $u_{2}, \cdots$ of elements of $R$ such that for $f$ in $R^{N},(M f)(n)=u_{0} f(n)+\cdots$ $+u_{n} f(0)$.

Consider a linear difference equation

$$
\begin{aligned}
& S_{1}(n-1) F(n-1)+\cdots+S_{k}(n-k) F(n-k) \\
& \quad=G(n)+T_{1}(n-1) G(n-1)+\cdots+T_{k}(n-k) G(n-k),
\end{aligned}
$$

where $S_{1}, \cdots, S_{k}, T_{1}, \cdots, T_{k}, F, G$ are functions on the set of rational integers (positive and negative) to $R$ which vanish for negative arguments. For fixed $S_{1}, \cdots, S_{k}, T_{1}, \cdots, T_{k}$, (4) induces a linear map $M: R^{N} \rightarrow R^{N}$ given by the requirement that whenever $F, G$ jointly satisfy (4), and $f$ is a member of $R^{N}$ such that $f(n)=F(n)$ for $n \geqq 0$, then $(M f)(n)=G(n)$ for $n \geqq 0$.

THEOREM 2. Let $R$ be a finite commutative ring with unit. Then $M: R^{N} \rightarrow R^{N}$ is a linear automaton transformation if and only if induced by a linear difference equation (4) with $S_{1}, \cdots, S_{k}, T_{1}, \cdots, T_{k}$ eventually periodic for $n \geqq 0$.

Corollary. Let $R$ be a finite commutative ring with unit. Then $M: R^{N} \rightarrow R^{N}$ is a translation invariant linear automaton transformation if and only if induced by a linear difference equation (4) with $S_{1}, \cdots$, $S_{k}, T_{1}, \cdots, T_{k}$ constant for $n \geqq 0$.

We will need three lemmas to prove Theorems 1 and 2 .

Lemma 1. Let $R$ be a finite commutative ring with unit. Endow $R$ with the discrete, $R^{N}$ with the product topology. Then $L: R^{N} \rightarrow R$ is linear and continuous if and only if there exists a finite sequence $W_{0}, \cdots, W_{m}$ of elements of $R$ such that for $f$ in $R^{N}, L f=W_{0} f(0)+\cdots$ $+W_{m} f(m)$.

Proof. It is an easy consequence of the compactness of $R^{N}$ and the continuity of $L$ that there exists an $m$ such that $L f_{1}=L f_{2}$ whenever $f_{1}, f_{2}$ are in $R^{N}$ and agree for $n \leqq m$. If we put $\delta_{k}(n)=1$ or 0 as $n=k$ or not, then we may take $W_{k}=L \delta_{k}$ for $k \leqq m$.

Call $M: A^{N} \rightarrow B^{N}$ causal if: for $f_{1}, f_{2}$ in $A^{N},\left(M f_{1}\right)(0)=\left(M f_{2}\right)(0)$; for $f_{1}, f_{2}$ in $A^{N}$ and $k>0$, if $f_{1}(n)=f_{2}(n)$ for $n<k$, then $\left(M f_{1}\right)(k)$ $=\left(M f_{2}\right)(k)$. Denote by $\sigma(A)$ the set of finite sequences $\left(x_{0}, \cdots, x_{j}\right)$ consisting of elements from a finite set $A$. Call two such sequences 
$\left(x_{0}, \cdots, x_{j}\right),\left(y_{0}, \cdots, y_{k}\right)$ state-equivalent (relative to $\left.M\right)$ if for any $f$ in $A^{N},\left(M f_{1}\right)(n+j+1)=\left(M f_{2}\right)(n+k+1)$ for all $n \geqq 0$, where $f_{1}, f_{2}$ are chosen satisfying: $f_{1}(n)=x_{n}$ for $0 \leqq n<j, f_{1}(n)=f(n-j)$ for $n \geqq j$, $f_{2}(n)=y_{n}$ for $0 \leqq n<k, f_{2}(n)=f(n-k)$ for $n \geqq k$. (Note that the stateequivalence of two sequences does not depend on the last member of either.) Define an intrinsic state for $M$ to be an equivalence class under state-equivalence.

Lemma 2. Let $A, B$ be finite nonempty sets. Then $M: A^{N} \rightarrow B^{N}$ is an automaton transformation if and only if $M$ is causal and $M$ possesses only a finite number of intrinsic states. Further, the least number of states required in order to induce $M$ as in (1) is the number of intrinsic states.

Proof. Suppose that $M$ is an automaton transformation. Then $M$ is certainly causal due to (1). We show that $M$ possesses no more intrinsic states than the number of elements of $Q$. If $X=\left(x_{0}, \cdots, x_{j}\right)$ is in $\sigma(A)$, define $q_{X}$ to be the $h(j)$ determined from (1) by letting $f(n)=x_{n}$ for all $n<j$. Then $X, Y$ in $\sigma(A)$ are state-equivalent whenever $q_{X}=q_{Y}$.

Conversely, if $M$ is causal and possesses only a finite set $Q$ of intrinsic states, define $\bar{b}, \bar{q}, M_{B}, M_{Q}$ as follows.

(i) Let $b=(M f)(0)$ for any $f$ in $A^{N}$.

(ii) Let $\bar{q}$ be the intrinsic state of any finite sequence of length 1 .

(iii) Let $M_{Q}\left(a, q_{1}\right)=q_{2}$ if for some $X$ in $q_{1}, Y$ in $q_{2}$, we have $X=\left(x_{0}, \cdots, x_{j}\right), Y=\left(y_{0}, \cdots, y_{j+1}\right), x_{n}=y_{n}$ for all $n<j, y_{j}=a$. Let $M_{B}\left(a, q_{1}\right)=(M f)(j+1)$ if $f$ is a member of $A^{N}$ such that $f(n)=y_{n}$ for $n \leqq j$.

Lemma 3. If $S_{1}, \cdots, S_{k}, T_{1}, \cdots, T_{k}$ are eventually periodic for $n \geqq 0$, then (4) induces a linear automaton transformation.

Proof. We wish to apply Lemma 2 ; it suffices to show that $M$ has only a finite number of intrinsic states, since any $M$ induced by Equation (4) is causal. Let $p_{i}, p_{i}^{\prime}$ be periods for $S_{i}, T_{i}, i=1, \cdots, k$. Then for $n_{1}$ sufficiently large, the intrinsic state of a finite sequence $\left(x_{0}, \cdots, x_{n+1}\right)$ is determined for $n \geqq n_{1}$ by $F(n-1), \cdots, F(n-k)$, $G(n-1), \cdots, G(n-k), n \bmod p_{1}, \cdots, n \bmod p_{k}, n \bmod p_{1}^{\prime}, \cdots$, $n \bmod p_{k}{ }^{\prime}$. Thus for $n \geqq n_{1}$, finite sequences fall into at most $z^{2 k} p_{1}$ $\cdots p_{s} p_{1}^{\prime} \cdots p_{s}^{\prime}$ distinct intrinsic states, where $z$ is the number of elements of $R$. Thus $M$ has altogether only a finite number of intrinsic states.

We now prove Theorems 1 and 2 . If $M$ is a linear automaton transformation, then for each $n \geqq 0$, the map $L_{n}: R^{N} \rightarrow R$ given by 
$L_{n} f=(M f)(n)$ is linear and continuous. Thus Lemma 1 applies and there exists a matrix $W_{n k}: N \times N \rightarrow R$ such that for each $n \geqq 0$ we can find an $m \geqq 0$ satisfying $(M f)(n)=W_{n 0} f(0)+\cdots+W_{n m} f(m)$, for all $f$ in $R^{N}$. Causality implies $W_{n k}=0$ for $k \geqq n$. Setting $u_{i j}=W_{(i+j) j}$ we need only verify (2) and (3) to satisfy Theorem 1.

(5) Suppose that $M: A^{N} \rightarrow B^{N}$ is an automaton transformation, and that $f$ is a member of $A^{N}$ such that $f(0), f(1), f(2), \cdots$ is eventually periodic. Then $(M f)(0),(M f)(1),(M f)(2), \cdots$ is eventually periodic. Moreover, if $q_{n}$ is the intrinsic state of $(f(0), \cdots, f(n))$, then $q_{0}, q_{1}, q_{2}, \cdots$ is eventually periodic.

We employ (5) to prove (2) and (3). Since the $k$ th column of $u_{i j}$ consists of the entries $0,\left(M \delta_{k}\right)(k+1),\left(M \delta_{k}\right)(k+2),\left(M \delta_{k}\right)(k+3), \cdots$ it follows that this column is completely determined by the intrinsic state of a $k$-term sequence consisting of $k-1$ zero entries followed by a one. Since this sequence has the same intrinsic state as a $k$-term sequence consisting of zeros, (5) applies to show that this intrinsic state is an eventually periodic function of $k$, and hence proves (3).

With this done, (2) is easy since it now suffices to show that the $k$ th column is itself eventually periodic. But (5) applied to $M \delta_{k}$ yields this.

Conversely, suppose that $M$ is defined by a matrix $u_{i j}$ satisfying (i), (ii), (iii) of Theorem 1. Define functions $U_{i}$ by $U_{i}(j)=u_{i j}$ for $j \geqq 0, U_{i}(j)=0$ for $j<0$. Then the following linear difference equation induces $M$ when recast in form (4). (In the notation of (2), put $k=p_{1}+P_{1}$.)

$$
\begin{aligned}
& U_{1}(n-1) F(n-1)+\cdots+U_{k}(n-k) F(n-k) \\
& \quad-U_{1}\left(n-p_{1}-1\right) F\left(n-p_{1}-1\right)-\cdots-U_{P_{1}}(n-k) F(n-k) \\
& \quad=G(n)-G\left(n-p_{1}\right) .
\end{aligned}
$$

By (3), $U_{1}, \cdots, U_{k}$ are eventually periodic for $n \geqq 0$; hence by Lemma $3, M$ is an automaton transformation. This proves both Theorem 1 and Theorem 2.

\section{REFERENCE}

1. S. C. Kleene, Representation of events in nerve nets and finite automata, Automata Studies, Princeton University Press, 1956, pp. 3-41.

Institute for Advanced Study 